\title{
The Minimum Number of Problems to Cover All Subproblems*
}

\author{
H. J. Greenberg**
}

(June 24, 1970)

\begin{abstract}
The following problem is motivated and then solved, using the theory of systems of distinct representatives. Let $M=\{1,2, \ldots, m\}$, and for each sequence $\sigma$ of distinct members of $M$, let $\langle\sigma\rangle$ be the associated subset. Suppose given a mathematical problem $P(S)$ for each subset $S$ of $M$, and an algorithm $A$ which when applied to $\sigma$ solves not only $P(\langle\sigma\rangle)$ but also all $P(\langle\tau\rangle)$ where $\tau$ is an initial segment of $\sigma$. What is the smallest number of applications of $A$ needed to solve the entire ensemble of problems $\{P(S): S \subset M\}$ ?
\end{abstract}

Key words: Combinatorics; dynamic programming; optimization; systems of distinct representatives; theory of computation.

\section{Introduction}

This note is presented with the same intent as a note by A. J. Goldman [4], ${ }^{1}$ where the motive is "to illustrate how mathematical disciplines that appear too abstruse to be useful to the operations researcher can in fact provide exactly what is needed to answer some of his questions." The particular mathematical discipline used in our analysis is the combinatorial theory of "distinct representatives."

Let $M=\{1,2, \ldots, m\}$, and suppose a mathematical problem $P(S)$ is associated with each subset $S$ of $M$. We shall be concerned with a measure of the computational labor required to solve the ensemble of problems $\{P(S): S \subset M\}$, under the especially favorable circumstances described below. Obtaining the ensemble of solutions will be referred to as covering all subproblems.

For any sequence $\sigma$ of distinct members of $M$, let $\langle\sigma\rangle$ denote the subset of $M$ whose members are the terms of $\sigma$. Suppose we have an algorithm $A$, which when applied to $\sigma$ solves not only the problem $P(\langle\sigma\rangle)$ but also all the problems $P(\langle\tau\rangle)$ where $\tau$ is an initial segment of $\sigma$. Our question is: What is the minimum number $N(\mathrm{~m})$ of applications of $A$ needed to cover all subproblems? To which sequences should $A$ be applied to achieve this minimum number?

The analysis of this question is deferred to the next section of the note. We first present several examples to show how this question arises in mathematical operations-research settings.

Example 1. Consider a 0-1 integer linear program with $m$ binary variables, and with objective function $c^{T} x$. There are $2^{m}$ binary $m$-vectors to consider. Methods of implicit enumeration $[1,3]$ evaluate the objective function for some subfamily of these $2^{m}$ arguments, and the efficiency of such a method is typically measured by comparing the size of this subfamily with the number $2^{m}$ of evaluations required in a brute-force approach.

We propose, however, that an appropriate basis for comparison may be not $2^{m}$ but rather the smaller value $N(m)$ defined above. To see why, observe that the binary $m$-vectors $x$ are in natural correspondence with the subsets $S$ of M; let $x(S)$ be the vector associated with $S$, and let $P(S)$ be the task of evaluating $c^{T} x(S)$. This evaluation involves, for some arrangement of the members of

\footnotetext{
AMS subject classification: Primary 05A05.

*An invited paper.

**Present address: Computer Sciences Center, Southern Methodist University, Dallas, Texas 75222.

${ }^{1}$ Figures in brackets indicate the literature references at the end of this paper.
} 
$S$ into a sequence $\sigma$, the formation of the successive partial sums $c_{\sigma(1)}, c_{\sigma(1)}+c_{\sigma(2)}$, etc. Thus we have an instance of the general question under study. The same idea applies when $P(S)$ is the evaluation of some function $F(S)$ which would naturally be calculated using a recursion

$$
F(T \cup\{j\})=F(T) * f(j) \quad(j \in M-T)
$$

where "*" is a commutative operation and $f$ is a function defined on $M$.

Example 2. As another example, suppose we have an $m$-stage dynamic program, the stages being numbered by the members of $M$. Assume this program has the special property that its solution is independent of the ordering of stages, and that the same is true of each of the subproblems $P(S)$ defined by using only some subset $S$ of the stages. For certain sensitivity analyses, it is desirable to solve the entire ensemble $\{P(S): S \subset M\}$ of problems; the recursive nature of the standard dynamic algorithm makes this an instance of the general question. For example, if $m=3$, it will follow from our analysis that the minimum number of dynamic programs which must be solved is 3 . One such trio is:

Program 1: Stage $1 \rightarrow$ Stage $2 \rightarrow$ Stage 3

Program 2: Stage $2 \rightarrow$ Stage 3

Program 3: Stage $3 \rightarrow$ Stage 1

Applying the dynamic programming algorithm to Program 1 yields the solutions of $P(\{1\})$, $P(\{1,2\})$, and $P(\{1,2,3\})$; application to Program 2 solves $P(\{2\})$ and $P(\{2,3\})$; and application to Program 3 yields the remaining nontrivial members $P(\{3\})$ and $P(\{1,3\})$ of the problem ensemble.

An example of a dynamic programming model having the stated commutivity property on its its stages is an additive resource allocation problem of the form:

$$
\begin{aligned}
\operatorname{Max} \sum_{j=1}^{m} f_{j}\left(x_{j}\right) & : \sum_{j=1}^{m} g_{j}\left(x_{j}\right) \leqslant b \\
& x_{j} \epsilon\left\{0,1, \ldots, d_{j}\right\}, \quad j=1, \ldots, n .
\end{aligned}
$$

The problem, $P(S)$, is of the form

$$
\begin{aligned}
\operatorname{Max} \sum_{j \in S} f_{j}\left(x_{j}\right): & \sum_{j \in S} g_{j}\left(x_{j}\right) \leqslant b \\
& x_{j} \epsilon\left\{0,1, \ldots, d_{j}\right\}, \quad j \in S .
\end{aligned}
$$

Example 3. The writer first encountered the general problem in the analysis of a parametric min-cut problem for communications networks [5]. In that context we are given a finite network with a set $N$ of nodes and a set $A$ of directed arcs; there is a distinguished source node $s$, and a distinguished subset $T$ of $N-\{s\}$ consisting of $m$ terminal nodes. A cut between $s$ and a subset, $T^{\prime}$, of $T$ is a subset of $A$ whose removal from the network would leave intact no path from the source $s$ to any node of $T^{\prime}$.

In this application we are also given a nonnegative rational-valued cost function $c$ defined on the set $A$ of arcs, and a nonnegative rational-valued value function $v$ defined on the set $T$ of terminals; $c$ is extended additively to the subsets of $A$ (e.g., cuts), and $v$ is extended additively to the subsets $T^{\prime}$ of $T$. The parametric analysis requires, for each number $V$ in the interval $[0, v(T)]$, determining among those subsets $T^{\prime}$ of $T$ with $v\left(T^{\prime}\right) \geqslant V$, one which minimizes $f\left(T^{\prime}\right)=\min [c(C)]$ where $C$ ranges over all cuts between $s$ and $T^{\prime \prime}$.

Such an analysis can be carried out in many ways [5]. The one which concerns us here involves the determination, for each of the $2^{m}$ subsets $T^{\prime}$ of $T$, of the associated value $f\left(T^{\prime}\right)$ (as well as the relatively trivial calculation of $v\left(T^{\prime}\right)$ ). Evaluation of a given $f\left(T^{\prime}\right)$ can be accomplished by 
applying the Ford-Fulkerson labelling algorithm [2]. This algorithm, however, has an inherent ability to solve subproblems in a recursive manner. That is, the "labels" determined in evaluating $f\left(T^{\prime}\right)$ can be used in solving for $f\left(T^{\prime} \cup\{t\}\right)$ where $t \epsilon T-T^{\prime}$. Thus, to determine the minimum number of explicit applications of the min-cut algorithm needed to complete the analysis while exploiting this recursive property, is again an instance of our general question.

\section{Analysis}

It is convenient to translate the general question into the setting of an abstract network, whose nodes are the $2^{m}$ subsets of $M$, and whose arcs are the pairs $\left(S, S^{\prime}\right)$ such that $S^{\prime}=S \cup\{j\}$ for some $j \epsilon M-S$. In this setting, an arrangement of the members of $S$ into a sequence $\sigma$ is equivalent to selecting a specific path from node $\phi$ (corresponding to the null set) to node $S$; initial segments of $\sigma$ correspond to initial subpaths of this path. Thus our general question takes the following form:

What is the minimum number, $N(m)$, of paths starting at $\phi$, which will cover all the nodes of this abstract network?

Let $[x]$ denote the greatest integer not exceeding $x$. It will be useful to have a special notation for the largest binomial coefficient with "upper" argument $m$; let

$$
g(m)=\left(\begin{array}{c}
m \\
{[m / 2]}
\end{array}\right) \text {. }
$$

Our analysis will yield a constructive proof that

$$
N(m)=g(m) .
$$

There are $g(m)$ nodes corresponding to $[\mathrm{m} / 2]$-tuples, and no two such nodes can be on a common path starting from $\phi$ (i.e., no $[\mathrm{m} / 2]$-tuple is contained in another). This shows that $N(m) \geqslant g(m)$. It only remains to show that there is a set of $g(m)$ paths starting at $\phi$ which together cover all nodes.

The abstract network has an obvious "mirror symmetry" associated with the one-to-one correspondence between sets $S$ and their complements $M-S$. Exploiting this, we see that it suffices to confine attention to the subnetwork containing the nodes corresponding to sets of cardinality $\leqslant[m / 2]$, and to show that all nodes of this subnetwork can be covered by a suitable set of $g(m)$ paths starting at $\phi$.

Let "level $p$ " refer to those nodes which correspond to subsets with $p$ members. The construction of the desired $g(m)$ paths can be effected recursively, proceeding from level to level in the network. It suffices to prove, by induction on $p$ for $p \leqslant[m / 2]$, that there are paths from $\phi$ to the $\left(\begin{array}{c}m \\ p\end{array}\right)$ nodes at level $p$, one path per node at level $p$, which are special in the sense that these paths together cover all nodes at levels $\leqslant p$. What is required for the induction step is to show, for $p<m / 2$, that one element can be deleted from each $(p+1)$-tuple in such a way that the resultant set of $p$-tuples includes all $p$-tuples. That is, there are one-arc extensions of the special paths for level $p$ (with each of these paths receiving at least one such extension) which together cover all nodes at level $p+1$; these extended paths are then clearly a special family for level $p+1$.

This is established in the following theorem and subsequent corollary, thus completing the proof of (2.1). The theorem will be demonstrated using the theory of distinct representatives, in particular the basic theorem of Hall [7]; an alternative would be the approach adopted by Meyers [8] in treating a combinatorial question somewhat related to that of the present note. 
Theorem: Let $\left\{\mathrm{T}_{\mathrm{i}}\right\}_{1}^{\mathrm{n}}$ be a collection of sets which collectively contain q elements, say $\left\{\mathbf{e}_{\mathrm{j}}\right\}_{1}^{\mathrm{a}}$. Let $\mathrm{T}_{\mathrm{i}}$ contain $\mathrm{t}_{\mathrm{i}}$ elements, and assume $\mathrm{e}_{\mathrm{j}}$ occurs in $\mathrm{r}_{\mathrm{j}}$ of the sets. Define

$$
\begin{aligned}
\rho & =\operatorname{Min}_{\mathrm{j}}\left\{\mathrm{r}_{\mathrm{j}}\right\}_{1}^{\mathrm{q}} \\
\tau & =\operatorname{Max}_{\mathrm{i}}\left\{\mathrm{t}_{\mathrm{i}}\right\}_{1}^{\mathrm{n}}
\end{aligned}
$$

If $\mathrm{q} \rho \geqslant \mathrm{n} \tau$ and $\rho \leqslant \tau$, then the family $\left\{\mathrm{T}_{\mathrm{i}}\right\}_{1}^{\mathrm{n}}$ has a SDR (system of distinct representatives).

Proof: Let $I$ be any index set from $\{1, \ldots, n\}$, with $k$ members. Suppose $\cup_{i \in I} T_{i}$ contains $a$ elements, say $\left\{e_{j}\right\}_{j \epsilon J}\left(J\right.$ is an index set from $\{1, \ldots, q\}$ with $a$ members). Then, for each $j \epsilon J^{c}$ (complement of $J$ ) none of the $r_{j}$ repetitions of $e_{j}$ are in any of $\left\{T_{i}: i \epsilon I\right\}$. Therefore, the number of appearances of elements in $\left\{T_{i}: i \in I^{c}\right\}$ is at least $\sum_{j \in J c} r_{j}$. This yields the inequality:

$$
\sum_{j \in J} r_{j} \leqslant \sum_{i \in I c} t_{i}
$$

Therefore,

$$
(q-a) \rho \leqslant(n-k) \tau
$$

Since $q \rho \geqslant n \tau$ and $\rho \leqslant \tau$, it follows that $a \geqslant k$. Our conclusion then follows from Hall's Theorem.

Observe that the counting process reveals $\sum_{j \in J^{c}} r_{j}=\sum_{i \in I^{c}} t_{i}$. Therefore, if all $r_{j}$ are equal and all $t_{i}$ are equal (so $r_{j}=\rho$ and $t_{i}=\tau$ ), and if $\rho \leqslant \tau$, then the theorem must apply with $q \rho=n \tau$. This says that in the corollary below we rely upon the fact that

instead of merely

$$
\left(\begin{array}{l}
m \\
p+1
\end{array}\right)(p+1)=\left(\begin{array}{l}
m \\
p
\end{array}\right)(m-p)
$$

$$
\left(\begin{array}{l}
m \\
p+1
\end{array}\right)(p+1) \geqslant\left(\begin{array}{l}
m \\
p
\end{array}\right)(m-p)
$$

Corollary: For the set $\mathrm{M}=\{1, \ldots, \mathrm{m}\}$, let $\left\{\mathrm{P}_{\mathrm{i}}\right\}_{1}^{\mathrm{k}}$ be the collection of all $\mathrm{p}$-tuples where $\mathrm{k}=\left(\begin{array}{c}\mathrm{m} \\ \mathrm{p}\end{array}\right)$ and $\mathrm{p}<\mathrm{m} / 2$. Then there exists a sequence of elements $\left\{\mathrm{s}_{\mathrm{i}}\right\}_{1}^{\mathrm{k}}$, with repetitions allowed, such that $\left\{\mathrm{P}_{\mathrm{i}} \cup \mathrm{s}_{\mathrm{i}}\right\}_{1}^{\mathrm{k}}$ are distinct $(\mathrm{p}+\mathrm{l})$-tuples.

Proof: Let $T_{i}$ be the collection of $m-p(p+1)$-tuples formed by adjoining to $P_{i}$ any member of its complement, $M-P_{i}$. Regard $P_{i}$ as "representable" by any $(p+1)$-tuple in $T_{i}$; then what is to be shown is that $\left\{T_{i}\right\}_{1}^{k}$ admits a system of distinct representatives.

In the notation of our theorem we have

$$
\begin{aligned}
q & =\left(\begin{array}{c}
m \\
p+1
\end{array}\right), \quad n=\left(\begin{array}{c}
m \\
p
\end{array}\right), \\
r_{j} & =p+1 \text { for all } j=1, \ldots, q, \\
t_{i} & =m-p \text { for all } i=1, \ldots, n .
\end{aligned}
$$

Therefore, $\rho=p+1$ and $\tau=m-p$. Observe that

$$
q \rho=n \tau \text { and } \rho \leqslant \tau \text { for } p<m / 2 .
$$

Hence, our proof is complete.

The computation of the $g(m)$ paths is performed level by level by solving for the completions (or deletions) indicated by the corollary. It suffices to consider $p<m / 2$ and describe a method to proceed from level $p$ to level $p+1$. In terms of our abstract network our problem is to fined a maxi- 
mal matching in the bipartite network consisting of the $\left(\begin{array}{c}m \\ p\end{array}\right)$ vertices of level $p$, the $\left(\begin{array}{c}m \\ p+1\end{array}\right)$ vertices of level $p+1$ and the edges that connect a node in level $p$ with a node in level $p+1$.

Equivalently, we can cast our problem as an assignment problem with a cost matrix, $C$, having only $0-1$ elements. We set $c_{i j}=0$ if node $i$ (in level $p$ ) is connected with node $j$ (in level $p+1$ ); otherwise $c_{i j}=1$. We then ask for an assignment of each row to a distinct column to minimize the sum total cost. We know there is a solution with zero cost, and the assignment provides the desired matching.

The author wishes to thank A. J. Goldman for his many helpful comments.

\section{References}

[1] Balas, E., An additive algorithm for solving linear programs with zero-one variables, Oper. Res. 13, 517-546 (1965).

[2] Ford, L. R., Jr., and Fulkerson, D. R., Flows in Networks (Princeton Univ. Press, Princeton, N.J., 1962).

[3] Glover, F., A multiphase-dual algorithm for the zero-one integer programming problem, Oper. Res. 13, 879-919 (1965).

[4] Goldman, A. J., Fractional container-loads and topological groups, J. Oper. Res. 16, 1218-1221 (1968).

[5] Greenberg, H. J., Optimal attack of a communications network, Ph. D. Thesis, The Johns Hopkins University (Baltimore, Md., 1968).

[6] Greenberg, H. J., The use of branching in dynamic programming for parametric analysis, Oper. Res. 15, 976-977 (1967).

[7] Hall, P., On representatives of subsets, J. London Math. Soc. 10, 26-30 (1935).

[8] Meyers, P. R., Minimum number of subsets to distinguish individual elements, J. Res. Nat. Bur. Stand. (U.S.), 71B (Math. and Math. Phys.), 21-22 (1967).

(Paper No. 74B4-335) 